\title{
Reasonable Disagreement and the Generally Unacceptable: A Philosophical Analysis of Making Fair Choices
}

\section{Benedict Rumbold}

School of Public Policy, UCL, UK.

b.rumbold@ucl.ac.uk

James Wilson

Department of Philosophy and Centre for Philosophy, Justice and Health, UCL, UK.

j.wilson@ucl.ac.uk

One of the most striking aspects of Making Fair Choices on the Path to Universal Health Coverage (WHO, 2014) is the willingness of its authors to make substantive ethical claims about resource allocation. Rather than merely seeking to identify fair processes to govern priority setting in healthcare, the report makes direct recommendations about the priorities that policy makers ought to endorse. This marks a significant break from much of the current literature on healthcare priority setting, which confines itself to procedural justice, rather than aiming to establish specific and substantive priority setting rules (Holm, 1998; cf. Ashcroft, 2008).

The report's return to substantive claims about justice in healthcare resource allocation is interesting not only because it swims against the philosophical tide but because it also appears implicitly to reject the reasoning that led one of the authors of the report, Norman Daniels, to advocate the retreat to procedural justice in the first place (Daniels and Sabin, 2008; Daniels, 2008, p. 109). In his seminal work on Accountability for Reasonableness, Daniels had argued that, given widespread scepticism about whether it is possible to reach a consensus on which substantive principles to use in healthcare resource allocation and how they should be specified and balanced against one another, we are forced to turn to questions of fair procedure (Daniels, 1994). According to Daniels, scepticism about substantive principles was justified: whilst some extreme positions can be ruled out as wrong, reasonable disagreement on topics such as when should we allow an aggregation of modest benefits to large numbers of people to outweigh more significant benefits to fewer people, or how much priority should we give to treating the sickest or most disabled 
patients, was taken to be unavoidable (at least for the foreseeable future) (Daniels, 2008, pp. 105-8).

In making substantive recommendations about how to resolve key priority setting disputes on the path to universal health coverage (UHC), then, the report seems to challenge this view, or at least question its scope. What the authors want to say, it seems, is that, when it comes to expanding coverage in health care, there are certain things about which we ought to agree (given certain substantive ethical arguments) and that such areas of consensus ought to form a basis for moving towards UHC, even though there will remain more fine grained choices that will need to be resolved on a country by country basis through the use of fair procedures.

There are two levels to the substantive ethical model that the report proposes. First, the report sets out how policy makers ought to sort interventions into high, medium and low priority. Here, despite some substantive recommendations, the report appears to give significant space to the possibility of reasonable disagreement. Thus, while the report stresses that whatever solution policy makers reach, if they are to follow good practice in an ethical sense, they need to include criteria relating to cost-effectiveness, (WHO, 2014, pp. 13-4), how far they benefit the worse-off (WHO, 2014, pp. 14-16), and whether the services whose coverage offers substantial financial risk protection (WHO, 2014, pp. 17-19). The report also notes that policy makers may wish to consider additional criteria as well as these three core considerations, such as severity of disease (present and future health gap), realization of potential, past health loss, criteria related to socioeconomic status, area of living and so on (WHO, 2014, pp. 19-20). Moreover, the report's authors make no claims as to how such values ought to be articulated and weighed against one another, arguing they should only be 'specified and balanced in a way that is sensitive to country context' (WHO, 2014, p. 23).

The second level of the report's substantive ethical model is more prescriptive. Here the authors outline five 'generally unacceptable trade-offs' (UTs) between two desirable goals as policy makers look to 'progressively realise' universal health care coverage. From earlier in the report, we know that 'progressive realisation' here covers three dimensions of change: i) extending current pooled funds to the non-covered, ii) reducing cost-sharing and fees, and iii) including other services (WHO, 2014, p. 5).

There are two primary features of the trade-offs that the authors deem generally unacceptable. First, trade-offs are unacceptable in the sense of being outside the bounds of, 
or in conflict with, good ethical practice in the pursuit of universal health coverage. As the report puts it, they are those that are 'incompatible with fair progressive realization of UHC' (WHO, 2014, p. 38). Second, the sense of 'good ethical practice' appealed to is a universal one. At various places the report acknowledges a certain degree of relativism or (perhaps) particularism about how the various trade-offs involved in realising UHC might play out; as the authors put it, ' $[t]$ hese decisions depend on context, and several different pathways can be appropriate' (WHO, 2014, p. ix). However, the report's recognition of certain trade-offs as 'unacceptable' comes in direct opposition to this, more pluralistic point of view; the point being that 'Nevertheless, some trade-offs are generally unacceptable' (our italics), which is to say, wrong irrespective of context (WHO, 2014, p. ix). In registering certain trade-offs as unacceptable, then, the report seems to be claiming that some trade-offs, in at least most circumstances, stand outside the bounds of proper ethical practice and therefore cannot form a part of a legitimate priority setting procedure in the realisation of UHC.

\section{Justifying the Unacceptable Tradeoffs}

What, then, justifies the view that certain trade-offs are ethically unacceptable in this strong sense? It seems clear that the unacceptability of a given trade-off is not supposed to follow simply and analytically from conclusions reached about the high-, medium- or low-priority of services at the first level of the report's substantive ethical model. The authors are explicit that the unacceptable trade-offs are only usually but not always unacceptable, and that what makes the difference is the other ethical values in play:

'There may be certain circumstances in which the generally unacceptable trade-offs are acceptable. First, there may be circumstances in which a policy involving a generally unacceptable trade-off will yield vastly greater total benefits, in terms of coverage or health improvement, than any other alternative policy. Second, there may be circumstances in which the worse off will be better off in absolute terms from a policy involving a generally unacceptable trade-off than from any other alternative policy.' (WHO, 2014, p. 37)

So it would seem that the ethical unacceptability of each of the UTs - and the circumstances in which the default rules against such trade-offs can be legitimately overturned - is to be derived through substantive ethical reasoning. However, this creates a tension: where the 
authors recuse themselves from making precise recommendations about choices at the first level of their substantive model -i.e. which interventions should fall into the categories of high, medium and low priority - they are willing to make strong claims about the ethical unacceptability of choices at the second level. Implicitly, the argument seems to be that where there can be reasonable disagreements about the number and weighting of the principles that are used to allocate interventions to different priority classes, there cannot be reasonable disagreement about certain trade-offs policy makers might make in expanding coverage once that prioritisation process has been carried out.

Occasionally, this tension can look to spill over into outright conflict. Consider UT2: 'to give high priority to very costly services whose coverage will provide substantial financial protection when the health benefits are very small compared to alternative, less costly services'. UT2 proscribes any attempt by policy makers to give 'high priority' to expensive, relatively cost-ineffective services (i.e. those that 'provide substantial financial protection' and with relatively small health benefit) over cheaper services (presumably) producing equal or greater benefits (and thus being necessarily more cost-effective). However, in making the claim that this trade-off 'generally unacceptable', the report appears to contradict its own attempts to stay neutral as to how the values of cost-effectiveness, benefit to the worse off and financial protection ought to be weighed against one another. According to UT2, in almost all cases cost-effectiveness should trump financial risk protection (WHO, 2014, p. 23).

Elsewhere, the report's insistence that certain trade-offs are ethically unacceptable (where various weightings between values at the first level of the model are not) also looks open to challenge. For example, take UT1: 'to expand coverage for low- or medium-priority services before there is near universal coverage for high-priority services,' which we are told, 'includes reducing out-of-pocket payments for low- or medium-priority services before eliminating out-of-pocket payments for high-priority services'. UT1 makes a claim about how policy-makers ought to prioritise (in terms of expanding coverage) between services already established as high-, medium- and low-priority within the given locality, the view being that investment ought to always be directed first to the highest-priority services. What UT1 seems to assert, then, is that before a country has completely covered the whole population, eliminated cost-sharing fees and introduced any services deemed high-priority, it is generally unacceptable for it to either extend current pooled funds to the non-covered, or to reduce cost-sharing and fees for any services deemed medium- or low-priority, or 
introduce a new medium or low priority service. One thing that immediately leaps out from this is a rather strong claim about relative moral importance of those values implicit in the ranking of health services (i.e. ensuring maximum benefit through cost-effectiveness, benefit to the worst off and so on) over those values implicit in the expansion of coverage (i.e. combating the injustices of non-coverage amongst the citizenship, financial barriers to access and inadequate service coverage). That is, on UT1, it is deemed generally unacceptable for a policy-maker to, say, extend current pooled funds to cover the currently non-covered for all those services the health system already provides (that is, high-, medium- and low-priority) before, say, growing the service package to include some further set of high-priority services, or reducing cost-sharing and fees for such services. Against the report's claim that these kinds of trade-offs are outside the bounds of good ethical practice, in Daniels's phrase, this kind of trade-off looks like something about which reasonable people may disagree. Certainly it does not seem to be 'unacceptable' for a policy-maker to seek to remove, say cultural or ethnic barriers to health care for all services (cost-effective and cost-ineffective), before seeking to ensure maximal coverage for cost-effective ones.

\section{Further Questions about the Unacceptable Tradeoffs}

There are other questions we might also ask of the UTs. For example: what is supposed to be the connection between them? That is, are they lexically ordered, or are we also supposed to balance their relative prescriptions against one another? This is particularly a problem when considering UT1 against UT3, ('to expand coverage for well-off groups before doing so for worse-off groups when the costs and benefits are not vastly different', which 'includes expanding coverage for those with already high coverage before groups with lower coverage'). UT3 requires policy makers to prioritise who they expand coverage for relative to how well-off they are, (with how well-off they are being made up, presumably of a number of factors including whether their current level of coverage, socio-economic status and so on), the prescription being that it is generally unacceptable to expand coverage for the well-off before the worse-off in circumstances where the costs and benefits are not vastly different. However, this seems to conflict with UT1. For example, what ought a policymaker to do when faced with the question of whether to expand coverage for low- and medium-priority services for the worse-off or expand coverage for high-priority services for the already well-off? Both options here, on different UTs, look generally unacceptable. We are left asking, then, which is the more unacceptable trade-off and why? 
Returning to the theme with which we began, there is also perhaps an unanswered question about how any of these trade-offs are to be balanced against the demands of procedural justice. That is, is a decision - for example to prioritise the availability of dialysis over other much more cost effective interventions - still generally unacceptable, even when made through a sound decision making procedure such as Daniels' Accountability for Reasonableness? At certain points the report seems to want to say that it is. However, one of Daniels' claims is that any decision made through Accountability for Reasonableness is both fair and legitimate.

Overall, we applaud the report's aims and indeed, many of its conclusions. However, we feel there is still much more that needs to be said in defence of its five unacceptable trade-offs: what they mean, why they genuinely are ethically unacceptable (where various choices made at the first level of the report's substantive ethical model are not), their various interrelationships and how they relate to matters of procedural justice. Given Making Fair Choices has been designed by its authors to appeal to a wide international audience, we do not fault the authors for failing to discuss these matters at length in the report itself. Rather, the points raised in this commentary are at most queries; we want to hear more. 


\section{References}

Ashcroft, R. (2008) 'Fair Process and the Redundancy of Bioethics: A Polemic', Public Health Ethics 1(1): 3-9.

Daniels, N. \& Sabin, J.E. (2008). Setting limits fairly: learning to share resources for health. $2^{\text {nd }}$ Edition. Oxford: Oxford University Press.

Daniels, N. (1994) 'Four Unsolved Rationing Problems: A Challenge', The Hastings Center Report, 24(4): 27-29.

Daniels, N. (2008) Just Health: Meeting Health Needs Fairly. Cambridge: Cambridge University Press.

Holm, S. (1998) 'Goodbye to the simple solutions: the second phase of priority setting in health care', BMJ 317:1000-2.

World Health Organisation (2014), Making fair choices on the path to universal health coverage. Geneva: World Health Organisation. Available at http://apps.who.int/iris/bitstream/10665/112671/1/9789241507158 eng.pdf?ua=1 - last accessed 27/07/14. 